\title{
RIGIDITY OF HYPERSURFACES OF CONSTANT SCALAR CURVATURE
}

\author{
BY C. E. HARLE ${ }^{1}$ \\ Communicated by P. E. Thomas, October 30, 1969
}

Let $M_{n}$ and $\tilde{M}_{n+1}$ be Riemannian manifolds of dimension $n$ and $n+1$ respectively.

Assume $M_{n}$ isometrically immersed in $\tilde{M}_{n+1}$.

If each point $p$ of $M_{n}$ is contained in an open neighborhood $U \subset M_{n}$ (which may depend on $p$ ) such that no open submanifold of $U$ is rigid in $\widetilde{M}_{n+1}$, then $M_{n}$ is called locally deformable in $\widetilde{M}_{n+1}$.

This concept allowed us to show that the result of NaganoTakahashi [3] holds without any restriction, i.e. that any homogeneous hypersurface of the Euclidean space $E_{n+1}$ is isometric to the Riemannian product of a $p$-dimensional sphere and an $n-p$ dimensional Euclidean space.

This result is a consequence of the following

THEOREM 1. Let $M_{n}$ be a complete Riemannian manifold of dimension $n \geqq 3$, with constant scalar curvature $K \neq 0$.

If $M_{n}$ is locally deformable in the Euclidean space $E_{n+1}$, then it is isometric to the Riemannian product of a 2-sphere of radius $1 / K$ and an $n-2$ dimensional Euclidean space.

The next result gives rigidity of certain hypersurfaces of nonEuclidean space forms.

THEOREM 2. Let $M_{n}$ be an n-dimensional Riemannian manifold $n \geqq 4$, with constant scalar curvature $K$. Assume $M_{n}$ isometrically immersed in the space form $\tilde{M}_{n+1}(c)$, with $c \neq 0$ and $K \neq n(n-1) c$. Then $M_{n}$ is rigid in $\tilde{M}_{n+1}(c)$.

\section{REFERENCES}

1. E. Cartan, La deformation des hypersurfaces dans l'espace reel a n-dimensions, Bull. Soc. Math. France 45 (1917) 57-121.

2. S. Dolbeaut-Lemoine, Sur la déformabilité des variétés plongées dans un espace de Riemann, Ann. Ecole Norm. Sup. (3) 73 (1956), 357-438. MR 18, 819.

3. T. Nagano and T. Takahashi, Homogeneous hypersurfaces in euclidean spaces, J. Math. Soc. Japan 12 (1960), 1-7. MR 22 \#5008.

Universidade de Sao Paulo, Sao Paulo, Brazil

AMS Subject Classifications. Primary 5374; Secondary 5370.

Key Words and Phrases. Hypersurfaces, homogeneous locally deformable, scalar curvature, Riemannian product, space form, sphere, Euclidean space.

1 This work has been supported by $\mathrm{CNP}_{q}$ (Rio de Janeiro, Brazil) and EPUSP (São Paulo, Brazil). 